\title{
Semiclassical quantization of non-Hermitian 2-D systems: Classical (Lie transform) Perturbation theory
}

\author{
Asiri Nanayakkara* \\ Institute of Fundamental Studies, Hanthana Road, Kandy.
}

Revised: 20 August 2008; Accepted: 24 November 2008

\begin{abstract}
Both real and complex semiclassical eigen energies of two dimensional non-Hermitian Hamiltonian systems are obtained by classical (Lie transform) perturbation theory requiring the action variables $I_{1}$ and $I_{2}$ to satisfy the quantization condition $I_{1}=\left(n_{1}+(1 / 2)\right) \hbar$ and $I_{2}=\left(n_{2}+(1 / 2)\right) \hbar$ respectively where $n_{1}, n_{2}$ are integers. Classical perturbation theory with Lie transform makes classical trajectories, which are non-periodic or non-quasi-periodic, periodic. It was observed that this method produces accurate eigen energies even when classical trajectories are not periodic or quasi-periodic. Eigen energies obtained by classical perturbation theory are compared with the same, determined by Rayleigh-Schroedinger perturbation theory
\end{abstract}

Keywords: Lie transform, non-Hermitian systems, semiclassical quantization

\section{INTRODUCTION}

Real non-separable multidimensional Harmonic Oscillator Systems have been widely used in the past for investigating classical chaos and its manifestation in quantum mechanics in Hamiltonian systems ${ }^{1-12}$. Further more, semiclassical investigations of these oscillators provide insight into the various experiments related to the intramolecular energy transfer and understanding of the nature and meaning of intramolecular randomization ${ }^{13}$. Although non-Hermitian multidimensional Harmonic oscillator systems do not provide such a practical insight yet, semiclassical investigations of nonHermitian complex Hamiltonian systems are important from both the fundamental and practical point of view. In the first place they may expose important clues hidden in the correspondence between classical and quantum mechanics, especially when classical motion becomes chaotic or PT symmetry of quantum states are spontaneously broken. Secondly, they provide powerful tools for calculations of highly excited energy spectra of the multidimensional complex systems. Particularly, when action variables or eigen energy expressions are available in analytic form, it is possible to have a better understanding on the classical behaviour, the semiclassical quantization and the semiclassical limit.

Classical perturbation theory based on Lie transforms has been used to obtain constants of motion of 2-D coupled Harmonic oscillator systems ${ }^{14,15}$ and to determine semiclassical eigen energies and linking quantum avoided crossing with classical chaos in real Hermitian systems in the past ${ }^{16,17}$. Classical motion of 1-D non-Hermitian Hamiltonian systems has been studied in several investigations ${ }^{1-21}$. In this paper, we use Lie transforms to investigate the classical frequencies and real and complex semiclassical eigen values of 2-D non Hermitian Hamiltonian systems analytically. In particular, we study two Hamiltonians, $H_{a}$ and $H_{b}$, which are non Hermitian and classical trajectories of $H_{b}$ which do not have periodic or quasi periodic trajectories,

$\mathrm{H}_{\mathrm{a}}=(1 / 2)\left[\mathrm{p}_{1}^{2}+\mathrm{p}_{2}{ }^{2}+\omega_{1}{ }^{2} x_{1}{ }^{2}+\omega_{2}{ }^{2} x_{2}^{2}\right]+\mathrm{ig} x_{1} x_{2}{ }^{2}$

and

$\mathrm{H}_{\mathrm{b}}=(1 / 2)\left[\mathrm{p}_{1}^{2}+\mathrm{p}_{2}^{2}+\omega_{1}^{2} x_{1}^{2}+\omega_{2}^{2} x_{2}^{2}\right]+\operatorname{igx}{ }_{1}^{2} x_{2}^{2} \cdots$

$H_{\mathrm{a}}$ is PT symmetric while $H_{\mathrm{b}}$ is neither PT symmetric nor pseudo-Hermitian. Using Lie transform, we find integrable Hamiltonians which are the integrable approximates to non-integrable Hamiltonians $H_{\mathrm{a}}$ and $H_{\mathrm{b}}$. Outline of the paper is as follows. In section I, we describe the classical perturbation theory (Lie transform) method and apply it for the above systems to obtain approximate integrable Hamiltonians. In section II, classical frequencies are 
obtained by solving the equation of motion in transformed coordinates. In section III, the semiclassical eigen values are calculated using classical perturbation theory and compares them with exact and Rayleigh-Schroedinger perturbation energies. Concluding remarks are given in section IV.

\section{Classical Lie canonical transform}

In this section, first we describe the classical perturbation theory based on Lie canonical transformations. (For further details, see reference $14 \& 15$ ). Consider the Hamiltonian of the form

$H=H_{0}+\varepsilon H_{1}$

where $\mathrm{H}_{0}=(1 / 2)\left[\mathrm{p}_{1}^{2}+\mathrm{p}_{2}^{2}+\omega_{1}^{2} x_{1}^{2}+\omega_{2}^{2} x_{2}^{2}\right]$ and $H_{1}\left(x_{1}, x_{2}\right)$ makes the above Hamiltonian $H$ non-separable and non-Hermitian. Hamilton's equation of motion for the above Hamiltonian is

$$
\frac{d p_{j}}{d t}=-\frac{\partial H}{\partial x_{j}}
$$

and

$$
\frac{d x_{j}}{d t}=\frac{\partial H}{\partial p_{j}}
$$

$(j=1,2)$.

Now consider the transformation $\left(p_{j}, x_{j}\right) \rightarrow\left(\eta_{j}, \xi_{j}\right)$ given by $p_{j}=\eta_{j}-\varepsilon \frac{\partial S_{1}}{\partial \xi_{j}}-\varepsilon^{2} \frac{\partial S_{2}}{\partial \xi_{j}}-\frac{1}{2} \varepsilon^{2}\left\{\frac{\partial S_{1}}{\partial \xi_{j}}, S_{1}\right\}+O\left(\varepsilon^{3}\right)$

and

$x_{j}=\xi_{j}+\varepsilon \frac{\partial S_{1}}{\partial \eta_{j}}-\varepsilon^{2} \frac{\partial S_{2}}{\partial \eta_{j}}-\frac{1}{2} \varepsilon^{2}\left\{\frac{\partial S_{1}}{\partial \eta_{j}}, S_{1}\right\}+O\left(\varepsilon^{3}\right)$

where \{\} represents the Poisson bracket and equations of motion become

$$
\begin{gathered}
\frac{d \eta_{j}}{d t}=-\frac{\partial H^{*}}{\partial \xi_{j}} \\
\frac{d \xi_{j}}{d t}=\frac{\partial H^{*}}{\partial \eta_{j}}
\end{gathered}
$$

$(j=1,2)$ and the new Hamiltonian $H^{*}(\eta, \xi)=H(p, x)$. The purpose for introducing this canonical transformation is, to eliminate, from Hamiltonian $H^{*}$, the parameter $\tau$ which is introduced in (11) and (12). Now we expand $H^{*}$ in a power series of $\varepsilon$ as

$\mathrm{H}^{*}(\eta, \xi)=\mathrm{H}_{0}^{*}(\eta, \xi)+\varepsilon \mathrm{H}_{1}^{*}(\eta, \xi)+$ and parameter $\tau$ is introduced by the auxiliary equation

$\frac{d \eta_{j}}{d \tau}=-\frac{\partial H_{0}^{*}}{\partial \xi_{j}}$

$\frac{d \xi_{j}}{d \tau}=\frac{\partial H_{0}^{*}}{\partial \eta_{j}}$

$(j=1,2)$ and hence $H_{0}{ }^{*}=$ const. The $H_{\mathrm{k}}{ }^{*} \mathrm{~s}$ in the power series (10) are obtained (details are given in reference 14,15 ) by

$$
\begin{aligned}
\mathrm{H}_{0}^{*} & =\left(\frac{1}{2}\right)\left[\eta_{1}{ }^{2}+\eta_{2}{ }^{2}+\omega_{1}{ }^{2} \xi_{1}{ }^{2}+\omega_{2}{ }^{2} \xi_{2}{ }^{2}\right] \\
H_{1}{ }^{*} & =\left[H_{1}(\eta, \xi)\right]_{s} \\
S_{1} & =\int\left[H_{1}(\eta, \xi)-\mathrm{H}_{1} *\right] d \tau \\
H_{2}{ }^{*} & =\frac{1}{2}\left\{H_{1}+H_{1}{ }^{*}, S_{1}\right\}_{s} \\
S_{2} & =\int\left[\frac{1}{2}\left\{H_{1}+H_{1}{ }^{*}, S_{1}\right\}-H_{2} *\right] d \tau \\
H_{3}{ }^{*} & =\frac{1}{2}\left\{H_{1}+H_{1}{ }^{*}, S_{2}\right\}_{s}+\frac{1}{2}\left\{H_{2}+H_{2}{ }^{*}, S_{1}\right\}_{s}+\frac{1}{12}\left\{\left\{H_{1}-H_{1}{ }^{*}, S_{1}\right\}, S_{1}\right\}_{s}
\end{aligned}
$$

where the subscript $s$ stands for the average value which is defined for a function $\mathrm{A}$ as

$\mathrm{A}_{\mathrm{s}}=\lim _{\mathrm{t} \rightarrow \infty} \frac{1}{\mathrm{t}} \int_{0}^{\mathrm{t}} \mathrm{A}(\tau) \mathrm{d} \tau$

and for a periodic function with period $T$ above equation becomes

$$
\mathrm{A}_{\mathrm{s}}=\frac{1}{\mathrm{~T}} \int_{0}^{\mathrm{T}} \mathrm{A}(\tau) \mathrm{d} \tau
$$

Now we obtain $\mathrm{H}_{0}{ }^{*}, \mathrm{H}^{*}$ and equivalent constants of motion for two non-Hermitian systems given in equations (1) and (2). The Hamiltonian $\mathrm{H}_{\mathrm{a}}$ in (1) is PT symmetric and known to have all eigen values real. Assuming $\omega_{1}$ is not commensurable with $\omega_{2}, H_{0}{ }^{*}(\eta, \xi)$ and $H^{*}(\eta, \xi)$ are found for the above system as

$H_{a}=\frac{1}{2}\left[\left(\xi_{1}^{2}+\eta_{1}^{2} / \omega_{1}^{2}\right) \omega_{1}^{2}+\left(\xi_{2}^{2}+\eta_{2}^{2} / \omega_{2}^{2}\right) \omega_{2}^{2}\right]-\frac{g^{2}}{2 \omega_{1}^{2}\left(\omega_{1}^{2}-4 \omega_{2}^{2}\right)} \beta_{a} \ldots$

where $\beta_{a}=\left\{\left(\omega_{2}{ }^{2}-3 \omega_{1}{ }^{2} / 8\right)\left(\xi_{2}{ }^{2}+\eta_{2}{ }^{2} / \omega_{2}{ }^{2}\right)^{2}+\omega_{1}{ }^{2}\left(\xi_{1}{ }^{2}+\eta_{1}{ }^{2} / \omega_{1}{ }^{2}\right)\right.$

$$
\left.\left(\xi_{2}{ }^{2}+\eta_{2}{ }^{2} / \omega_{2}{ }^{2}\right)\right\}
$$

$H_{a 0} *=\frac{1}{2}\left(\eta_{1}^{2}+\eta_{2}^{2}+\omega_{1}^{2} \xi_{1}^{2}+\omega_{2}^{2} \xi_{2}^{2}\right.$

This new system admits two first integrals, $H_{a 0}{ }^{*}=$ const and $H_{a}^{*}=$ const and they are equivalent to

$\left(\xi_{1}^{2}+\eta_{1}^{2} / \omega_{1}^{2}\right)=$ const 
$\left(\xi_{2}^{2}+\eta_{2}^{2} / \omega_{2}^{2}\right)=$ const

Above equations (16), (18) and (19) will be used to quantize this system in the section III. Now we find similar expressions of $H_{0}{ }^{*}$ and $H^{*}$ for the Hamiltonian systems $H_{b}$.

$$
\begin{aligned}
H_{b}= & \frac{1}{2}\left[\left(\xi_{1}^{2}+\eta_{1}^{2} / \omega_{1}^{2}\right) \omega_{1}^{2}+\left(\xi_{2}^{2}+\eta_{2}^{2} / \omega_{2}^{2}\right) \omega_{2}^{2}\right]+ \\
& \frac{1}{4} \frac{\left(\xi_{1}^{2}+\eta_{1}^{2} / \omega_{1}^{2}\right)\left(\xi_{2}^{2}+\eta_{2}^{2} / \omega_{2}^{2}\right)}{\omega_{1}^{2} \omega_{2}^{3}\left(\omega_{2}^{2}-\omega_{1}^{2}\right)}\left\{i g+\frac{g^{2}}{2} \beta\right\} \cdots
\end{aligned}
$$

where

$$
\begin{aligned}
& \beta=\left\{\left(3 \omega_{2}^{2}-2 \omega_{1}^{2}\right)\left(\xi_{1}^{2}+\eta_{1}{ }^{2} / \omega_{1}{ }^{2}\right)+\left(3 \omega_{1}{ }^{2}-2 \omega_{2}{ }^{2}\right)\left(\xi_{2}{ }^{2}+\eta_{2}{ }^{2} / \omega_{2}{ }^{2}\right)\right\} \\
& H_{b 0}=\frac{1}{2}\left(\eta_{1}^{2}+\eta_{2}^{2}+\omega_{1}^{2} \xi_{1}^{2}+\omega_{2}^{2} \xi_{2}^{2}\right)
\end{aligned}
$$

The above Hamiltonians are functions of new variables $\xi_{1}, \xi_{2}, \eta_{1}$, and $\eta_{2}$ only in the form $\xi_{1}{ }^{2}+\eta_{1}{ }^{2} / \omega_{1}{ }^{2}$ and $\xi_{2}{ }^{2}+\eta_{2}{ }^{2}$ $\omega_{2}{ }^{2}$. These transformed Hamiltonians are merely the Borkhoff normal forms ${ }^{1,22}$ of the original Hamiltonians.

\section{Classical frequencies}

In the previous section we expressed the Hamiltonians of two non-Hermitian systems given by (1) and (2) in terms of constants of motion using classical Lie transform method. Constants of motion for these two dimensional potentials, are

$\left(\xi_{1}^{2}+\eta_{1}^{2} / \omega_{1}^{2}\right)=$ const $-c_{1}$

$\left(\xi_{2}^{2}+\eta_{2}^{2} / \omega_{2}^{2}\right)=$ const $-c_{2}$

Therefore, functions of $\xi_{1}{ }^{2}+\eta_{1}{ }^{2} / \omega_{1}{ }^{2}$ and $\xi_{2}{ }^{2}+\eta_{2}{ }^{2} / \omega_{2}{ }^{2}$ are also constants of motion of those systems. In terms of $c_{1}$ and $\mathrm{c}_{2}$, Hamiltonians $\mathrm{H}_{\mathrm{a}}$ and $\mathrm{H}_{\mathrm{b}}$ become

$$
\begin{gathered}
H_{a} *=\frac{1}{2}\left[c_{1} \omega_{1}^{2}+c_{2} \omega_{2}^{2}\right]-\frac{g^{2}\left\{\left(\omega_{2}^{2}-3 \omega_{1}^{2} / 8\right) c_{2}^{2}+\omega_{1}^{2} c_{1} c_{2}\right.}{2 \omega_{1}^{2}\left(\omega_{1}^{2}-4 \omega_{2}^{2}\right)} \cdots \\
H_{b}{ }^{*}=\frac{1}{2}\left[c_{1} \omega_{1}^{2}+c_{2} \omega_{2}^{2}\right]+\frac{1}{4} \frac{c_{1} c_{2}\left\{i g+g^{2}\left\{\left(3 \omega_{2}^{2}-2 \omega_{1}^{2}\right) c_{1}+\left(3 \omega_{1}^{2}-2 \omega_{2}^{2}\right) c_{2}\right\} / 2\right\}}{\omega_{1}^{2} \omega_{2}^{3}\left(\omega_{2}^{2}-\omega_{1}^{2}\right)}
\end{gathered}
$$

respectively. Using equation (8) and (9), we write the equations of motion for the above systems in terms of $c_{1}$ and $c_{2}$ as

$$
\frac{d^{2} \xi_{j}}{d t^{2}}+\Omega_{k, j}^{2} \xi_{j}=0
$$

where $k=\{a, b\}, j=\{1,2\}$ and $\Omega_{k, j}=\frac{2}{\omega_{j}}\left(\frac{\partial H_{k} *}{\partial c_{j}}\right)$. Frequencies $\Omega_{\mathrm{k}, \mathrm{j}}$ are independent of time as $\mathrm{c}_{1}$ and $\mathrm{c}_{2}$ are constants of motion. Therefore, the solutions of equation motion (26) are simply

$$
\begin{aligned}
& \xi_{j}=\sqrt{c_{j}} \operatorname{Cos}\left(\Omega_{k, j} t+\varphi\right) \\
& \eta_{j}=-\Omega_{k, j} \sqrt{c_{j}} \operatorname{Sin}\left(\Omega_{k, j} t+\varphi\right)
\end{aligned}
$$

where $\varphi$ is an integration constant and

$$
\begin{aligned}
& \Omega_{a, 1}=\omega_{1}-\frac{g^{2} c_{2}}{\omega_{1}\left(\omega_{1}^{2}-4 \omega_{2}^{2}\right)} \\
& \Omega_{a, 2}=\omega_{2}-\frac{2 g^{2} c_{2}\left(\omega_{2}^{2}-3 \omega_{1}^{2} / 8\right)}{\omega_{2} \omega_{1}^{2}\left(\omega_{1}^{2}-4 \omega_{2}^{2}\right)}-\frac{g^{2} c_{1}}{\omega_{2}\left(\omega_{1}^{2}-4 \omega_{2}^{2}\right)} \ldots(28) \\
& \Omega_{b, 1}=\omega_{1}+\frac{1}{2} \frac{c_{2}\left\{i g+\frac{g^{2}}{2}\left\{\left(3 \omega_{2}^{2}-2 \omega_{1}^{2}\right) c_{1}+\left(3 \omega_{1}^{2}-2 \omega_{2}^{2}\right) c_{2}\right\}\right\}}{\omega_{1}^{3} \omega_{2}^{3}\left(\omega_{2}^{2}-\omega_{1}^{2}\right)}+\frac{c_{1} c_{2} g^{2}\left(3 \omega_{2}^{2}-2 \omega_{1}^{2}\right)}{4 \omega_{1}^{3} \omega_{2}^{3}\left(\omega_{2}^{2}-\omega_{1}^{2}\right)} \\
& \Omega_{b, 2}=\omega_{2}+\frac{1}{4} \frac{c_{1}\left\{i g+\frac{g^{2}}{2}\left\{\left(3 \omega_{2}^{2}-2 \omega_{1}^{2}\right) c_{1}+\left(3 \omega_{1}^{2}-2 \omega_{2}^{2}\right) c_{2}\right\}\right\}}{\omega_{1}^{2} \omega_{2}^{4}\left(\omega_{2}^{2}-\omega_{1}^{2}\right)}+\frac{c_{1} c_{2} g^{2}\left(3 \omega_{2}^{2}-2 \omega_{1}^{2}\right)}{4 \omega_{1}^{2} \omega_{2}^{4}\left(\omega_{2}^{2}-\omega_{1}^{2}\right)}
\end{aligned}
$$

It is important to note that Hamiltonians $H_{k}^{*}(k=\{a, b\})$ are integrable, while corresponding original Hamiltonians $H_{k}$ are not. $H_{k}{ }^{*}$ are the integrable approximates to $H_{k}$. It is evident from the above expressions for the frequencies of oscillations $\Omega_{k, j}$, that the classical trajectories of the first Hamiltonian system is periodic with real frequencies. However, the frequencies of the second Hamiltonian $H_{b}{ }^{*}$ are complex and classical trajectories of that Hamiltonian are therefore non-periodic. Under the Lie transform, non-integrable Hamiltonians became integrable and quasi-periodic trajectories of the first pseudo Hermitian Hamiltonian became periodic. However, trajectories of the second non-pseudo Hermitian Hamiltonians did not become periodic. Then an important question to address is that whether the semiclassical quantization of the above complex non-Hermitian systems accurately produce the quantum eigen values as it does in the case of real Hermitian systems. This issue is addressed in the next section.

\section{Semiclassical eigen values}

In order to find semiclassical energy eigen values of those three Hamiltonians, first we make the transformation to action variables as or $\mathrm{I}_{\mathrm{j}}=\left(\xi_{\mathrm{j}}^{2}+\eta_{\mathrm{j}}^{2} / \mathrm{w}_{\mathrm{j}}^{2}\right) \mathrm{w}_{\mathrm{j}} \pi$

$I_{1}=\left(\xi_{1}^{2}+\eta_{1}^{2} / \omega_{1}^{2}\right) \omega_{1} \pi$

$I_{1}=\left(\xi_{2}^{2}+\eta_{2}^{2} / \omega_{2}^{2}\right) \omega_{2} \pi$ 
Therefore, Hamiltonians in terms of action variable $I_{1}$ and $I_{2}$ are given by

$$
\begin{aligned}
H^{a b}\left(I_{1}, I_{2}\right)= & \frac{1}{2 \pi}\left[I_{1} \omega_{1}+I_{2} \omega_{2}\right]-\frac{2 g^{2}}{\pi^{2} \omega_{1}^{2} \omega_{2}^{2}\left(\omega_{1}^{2}-4 \omega_{2}^{2}\right)} \beta \ldots \\
H^{b}\left(I_{1}, I_{2}\right)= & \frac{1}{2 \pi}\left[I_{1} \omega_{1}+I_{2} \omega_{2}\right]+ \\
& \frac{I_{1} I_{2}}{\pi^{3}\left(\omega_{2}^{2}-\omega_{1}^{2}\right) \omega_{1}^{3} \omega_{2}^{4}}\left\{i g+\frac{g^{2}}{\omega_{1} \omega_{2}} \beta_{b}, \ldots\right.
\end{aligned}
$$

where $\beta_{a}=\left\{\left(\omega_{2}^{2}-3 \omega_{1}{ }^{2} / 8\right) I_{2}{ }^{2}+I_{1} I_{2} \omega_{1} \omega_{2}\right\}$ and

$$
\beta_{b}=\left\{\left(3 \omega_{2}{ }^{2}-2 \omega_{1}{ }^{2}\right) I_{1} \omega_{2}+\left(3 \omega_{1}{ }^{2}-2 \omega_{2}{ }^{2}\right) I_{2} \omega_{1}\right\}
$$

Now we quantize the systems $H_{a}$ and $H_{b}$ with the quantization conditions

$I_{1}=\left(n_{1}+\frac{1}{2}\right) \hbar$

$I_{2}=\left(n_{2}+\frac{1}{2}\right) \hbar$

where $n_{1}$ and $n_{2}$ are the two quantum numbers. In this way, we can quantize both non-Hermitian Hamiltonians discussed above. Now we derive expressions for quantum mechanical eigen energies by applying aforementioned quantization conditions.

Imposing conditions (34) and (35) on (32) and (33), we obtain the quantum eigen energy expressions for the Non-Hermitian systems defined in (1) and (2) respectively as

$$
\begin{aligned}
E^{a}{ }_{n_{1} n_{2}}= & {\left[\left(n_{1}+\frac{1}{2}\right) \omega_{1}+\left(n_{2}+\frac{1}{2}\right) \omega_{2}\right]-} \\
& \frac{2 g^{2}}{\omega_{1}^{2} \omega_{2}^{2}\left(\omega_{1}^{2}-4 \omega_{2}^{2}\right)} \beta_{a} \\
E^{b}{ }_{n_{1} n_{2}}= & {\left[\left(n_{1}+\frac{1}{2}\right) \omega_{1}+\left(n_{2}+\frac{1}{2}\right) \omega_{2}\right]+} \\
& \frac{\left(n_{1}+\frac{1}{2}\right)\left(n_{2}+\frac{1}{2}\right)}{\omega_{1}^{3} \omega_{2}^{4}\left(\omega_{2}^{2}-\omega_{1}^{2}\right)}\left\{i g+\frac{g^{2}}{\omega_{1} \omega_{2}} \beta_{b}\right\}
\end{aligned}
$$

where now $\beta_{a}=\left\{\left(\omega_{2}{ }^{2}-3 \omega_{1}{ }^{2} / 8\right)\left(n_{2}+1 / 2\right)^{2} \omega_{1}{ }^{2}+\left(n_{1}+1 / 2\right)\right.$ $\left(n_{2}+1 / 2\right) \omega_{1} \omega_{2}$ and $\beta_{b}=\left\{\left(3 \omega_{2}{ }^{2}-2 \omega_{1}{ }^{2}\right)\left(n_{1}+1 / 2\right) \omega_{2}+\left(3 \omega_{1}{ }^{2}-\right.\right.$ $\left.\left.2 \omega_{2}^{2}\right)\left(n_{2}+1 / 2\right) \omega_{1}\right\}$ and $\hbar$ is taken as unity.
$E_{n_{1} n_{2}}^{a}$ and $E_{n_{1} n_{2}}^{b}$ in (36) and (37) are the quantum eigen energies of the systems (1) and (2) respectively and $n_{1}$ and $n_{2}$ are non negative integers (quantum numbers). Tables 1 and 2 contain the eigen energies calculated using second order Lie transform method and the second order Rayleigh-Schroedinger perturbation theory. For comparison purposes, exact eigen energies which are obtained by diagonalizing the Hamiltonian in Harmonic oscillator basis set are also included in each table.

Table 1: First ten eigen energies of $\mathrm{V}(x)=(1 / 2)\left[\omega_{1}{ }^{2} x_{1}{ }^{2}+\omega_{2}{ }^{2} x_{2}{ }^{2}+\operatorname{igx} x_{1} x_{2}^{2}\right.$ where $\omega_{1}=0.7, \omega_{2}=1.3$ and $g=0.08$ are calculated using Lie transform method and the second order Rayleigh-Schroedinger (RS) perturbation theory. "Exact" eigen energies are obtained by diagonalizing the Hamiltonian in Harmonic oscillator basis set.

\begin{tabular}{ccc}
\hline Exact & Lie transform & $2^{\text {nd }}$ order RS Perturb \\
\hline 1.00137 & 1.00149 & 1.00138 \\
1.70247 & 1.70261 & 1.7025 \\
2.30981 & 2.31004 & 2.30992 \\
2.40355 & 2.40373 & 2.40362 \\
3.01305 & 3.0134 & 3.01329 \\
3.10462 & 3.10485 & 3.10474 \\
3.62548 & 3.62601 & 3.6259 \\
3.71624 & 3.71677 & 3.71665 \\
3.80567 & 3.80598 & 3.80586 \\
4.33079 & 4.33162 & 4.33151 \\
\hline
\end{tabular}

Table 2: First ten eigen energies $V(x)=(1 / 2)\left[\omega_{1}^{2} x_{1}{ }^{2}+\omega_{2}{ }^{2} x_{2}{ }^{2}+\operatorname{ig} x_{1} x_{2}{ }^{2}\right.$ of where $\omega_{1}=0.7, \omega_{2}=1.3$ and $g=0.05$ are calculated using Lie transform method and Rayleigh-Schroedinger (RS) perturbation theory. "Exact" eigen energies are obtained by diagonalizing the Hamiltonian in Harmonic oscillator basis set.

\begin{tabular}{ccc}
\hline Exact & Lie Transform & $2^{\text {nd }}$ order RS Perturb \\
\hline $1.0006+0.0137 i$ & $1.0005+0.0137 i$ & $1.0006+0.0137 i$ \\
$1.7026+0.0408 i$ & $1.7029+0.0412 i$ & $1.7027+0.0412 i$ \\
$2.3033+0.0406 i$ & $2.3027+0.0412 i$ & $2.3034+0.0412 i$ \\
$2.4064+0.0674 i$ & $2.4073+0.0687 i$ & $2.4067+0.0687 i$ \\
$3.0121+0.1210 i$ & $3.0125+0.1236 i$ & $3.0129+0.1236 i$ \\
$3.1117+0.0930 i$ & $3.1136+0.0962 i$ & $3.1128+0.0962 i$ \\
$3.6074+0.0669 i$ & $3.6066+0.0687 i$ & $3.6079+0.0687 i$ \\
$3.7258+0.1992 i$ & $3.7282+0.2060 i$ & $3.7283+0.2060 i$ \\
$3.8181+0.1174 i$ & $3.8219+0.1236 i$ & $3.8208+0.1236 i$ \\
$4.3255+0.1993 i$ & $4.3272+0.2060 i$ & $4.3283+0.2060 i$ \\
\hline
\end{tabular}

The above two tables clearly show that the semiclassical eigen energies are in good agreement with exact quantum eigen values regardless of the Hermiticity of the Hamiltonians and whether the periods of the trajectories 
are real or not. For the Hamiltonian $H_{b}$, the second order Rayleigh-Schroedinger (RS) perturbation theory produces same complex energies as the semiclassical energies calculated with Lie transform method.

\section{Concluding remarks}

In this paper, we studied two 2D non Hermitian complex Hamiltonians, $H_{a}$ and $H_{\mathrm{b}}$ with Classical Lie transform method. The first Hamiltonian $H_{a}$ is PT symmetric and the entire quantum energy spectrum is found to be real and positive. In this study it was found that classical phase space of $H_{a}$ contains periodic trajectories and analytic expression for frequency of oscillations was obtained. Semiclassical energies are obtained as an algebraic expression and semiclassical eigen energies are found to be in very good agreement with exact quantum eigen energies.

The second Hamiltonian $H_{\mathrm{b}}$ is not even pseudo Hermitian. The entire quantum energy spectrum is complex. The classical frequencies are found to be complex and hence all the classical trajectories are non periodic. Since an action variable is intrinsically a concept associated with periodic motion, existence of action variable is directly linked with that of the periodic motion. However, semiclassical eigen energies based on quantization of the action variables in this system are found to be quite accurate although no trajectories of this system is periodic.

\section{References}

1. Siegel C.L. \& Moser J.K. (1971). Lectures on Celestial Mechanics, Springer-Verlag, Berlin, Germany.

2. Arnold V.I. \& Avez A. (1968). Ergodic Problems of Classical Mechanics. $1^{\text {st }}$ Edition, pp. 286. W.A. Benjamin Company, New York, USA.

3. Noid D., Koszykowski M., Tabor M. \& Marcus R. (1980). Properties of vibrational energy levels in the quasi periodic and stochastic regimes. Journal of Chemical Physics 72: 6169.

4. Noid D., Koszykowski M. \& Marcus R.A. (1983). Comparison of quantal, classical, and semiclassical behaviour at an isolated avoided crossing. Journal of Chemical Physics 78: 4018.

5. Uzer T., Noid D. \& Marcus R. (1983). Uniform semiclassical theory of avoided crossings. Journal of Chemical Physics 79: 4412.
6 Farrelly D. \& Uzer T. (1986). Semiclassical quantization of slightly nonresonant systems: avoided crossings, dynamical tunneling and molecular spectra. Journal of Chemical Physics 85: 308.

7. Takami T.(1995). Semiclassical study of avoided crossings. Physical Review E 52(3): 2434.

8. Brumer P. \& Duff J. (1976). A variational equations approach to the onset of statistical intramolecular energy transfer. Journal of Chemical Physics 65: 3566.

9. Cejan C. \& Reinhardt W. (1979). Critical point analysis of instabilities in Hamiltonian systems: classical mechanics of stochastic intramolecular energy transfer. Journal of Chemical Physics 71: 1819.

10. Stratt R., Handy N. \& Miller W. (1979). On the quantum mechanical implications of classical ergodicity. Journal of Chemical Physics 71: 3311.

11. Weissman Y. \& Jortner J. (1982). Quantum manifestations of classical stochasticity: I. energetics of some nonlinear systems. Journal of Chemical Physics 77: 1469.

12. Weissman Y. \& Jortner J. (1982). Quantum manifestations of classical stochasticity: II. dynamics of wave packets of bound states. Journal of Chemical Physics 77: 1486.

13. Noid D., Koszykowski M. \& Marcus R. (1981). Quasiperiodic and stochastic behavior in molecules. Annual Review of Physical Chemistry 32: 267.

14. Hori G. (1966). Theory of general perturbations with unspecified cannonical variables. Publications of the Astronomical Society of Japan 18(4): 287.

15. Hori G. (1967). Non-linear couplings of two harmonic oscillations. Publications of the Astronomical Society of Japan 19(2): 229.

16. Ramaswamy R. \& Marcus R. (1981). On the onset of chaotic motion in deterministic systems. Journal of Chemical Physics 74(2): 1385.

17. Ramaswamy R., Siders P. \& Marcus R. (1980). Semiclassical quantization of multidimensional systems. Journal of Chemical Physics 73(10): 5400.

18. Nanayakkara A. (2004). Classical trajectories of 1D complex non-Hermitian Hamiltonian systems. Journal of Physics A: Mathematics and General 37: 4321.

19. Bender C.M., Boettcher S. \& Meisinger P.N. (1999). PT-symmetric quantum mechanics. Journal of Mathematical Physics 40: 2201.

20. Bender C.M., Chen J., Darg D.W. \&. Milton K.A. (2006). Classical trajectories for complex Hamiltonians. Journal of Physics A: Mathematics and General 39: 4219.

21. Bender C.M. \& Darg D.W. (2007). Spontaneous breaking of classical PT symmetry. Journal of Mathematical Physics 48: 042703.

22. Birkhoff G.D. (1922). Surface transformations and their dynamical applications. Acta Mathematica 43: 1. 